ANNALES

POLONICI MATHEMATICI

$80(2003)$

\title{
On the removal of subharmonic singularities of plurisubharmonic functions
}

\author{
by E. M. Chirka (Moscow) \\ Dedicated to Prof. J. Siciak on the occasion of his 70th birthday
}

\begin{abstract}
It is proved that any subharmonic function in a domain $\Omega \subset \mathbb{C}^{n}$ which is plurisubharmonic outside of a real hypersurface of class $C^{1}$ is indeed plurisubharmonic in $\Omega$.
\end{abstract}

1. Let $E$ be a closed nowhere dense subset of a domain $\Omega$ in $\mathbb{C}^{n}$ and $u$ be a subharmonic function in $\Omega$, which is plurisubharmonic (psh) in $\Omega \backslash E$. The question is what conditions on $E$ guarantee the plurisubharmonicity of $u$ in the whole domain $\Omega$. For the question to be nontrivial, we consider the sets $E$ which are nonremovable for general bounded psh functions in $\Omega \backslash E$. The simplest class of such singularities is given by smooth hypersurfaces in $\Omega$, so the following theorem can be considered as a first step towards the solution of the general problem.

ThEOREM. Let $\Gamma$ be a $C^{1}$-hypersurface in a domain $\Omega \subset \mathbb{C}^{n}$ and $u$ be a subharmonic function in $\Omega$ which is plurisubharmonic in $\Omega \backslash \Gamma$. Then $u$ is plurisubharmonic in $\Omega$.

Note that we do not assume any smoothness of $u$ in $\Omega$. If $\Gamma$ divides $\Omega$ into two components $\Omega_{ \pm}$and $u \in C^{2}(\Omega \backslash \Gamma) \cap C^{1}\left(\bar{\Omega}_{ \pm}\right)$is continuous in $\Omega$, the condition of subharmonicity of $u$ in $\Omega$ means that $\Delta u \geq 0$ in $\Omega \backslash \Gamma$ and $\partial u / \partial n_{+}+\partial u / \partial n_{-} \geq 0$ on $\Gamma$ where $\partial u / \partial n_{ \pm}$are the (inner) normal derivatives of $u \mid \Omega_{ \pm}$at points of $\Gamma$. This case of "classical" smoothness of $u$ was considered by P. Blanchet [1] who proved the theorem under these additional assumptions. The general case needs another technique, even for $C^{\infty}$-hypersurfaces and $u$ piecewise smooth. On the other hand, our proof

2000 Mathematics Subject Classification: 32U30, 32U40.

Key words and phrases: plurisubharmonic function, removal of singularities.

Research supported by the Russian FBR, grant 02-01-01291. 
does not work already in the case of Lipschitz graphs (the $C^{1}$-smoothness of $\Gamma$ is essential). The following question remains open:

Let $E$ be a closed subset of $\Omega \subset \mathbb{C}^{n}$ with locally finite Hausdorff $(2 n-1)$ measure and let a function $u$ be subharmonic in $\Omega$ and psh in $\Omega \backslash E$. Does it follow that $u$ is plurisubharmonic in $\Omega$ ?

2. The proof of the theorem is based on the notion of positive currents (see [3, 2]). Recall that $v \in \operatorname{psh}(\Omega)$ if and only if the current $d d^{c} v=$ $i \sum v_{j k} d z_{j} \wedge d \bar{z}_{k}$ of bidegree $(1,1)$ in $\Omega$ is positive, that is, $\left(d d^{c} v, \Phi\right) \geq 0$ for each positive $(n-1, n-1)$-form $\Phi$ of class $C_{0}^{\infty}(\Omega)$. (Here $d^{c}=i(\bar{\partial}-\bar{\partial})$; the class of test forms $\Phi$ can be reduced to $\Phi$ of the type $\varphi \prod_{\nu=1}^{n-1}\left(i d l_{\nu} \wedge d \bar{l}_{\nu}\right)$ where $\varphi \in C_{0}^{\infty}(\Omega), \varphi \geq 0$ and $l_{\nu}$ are $\mathbb{C}$-linear functions in $\mathbb{C}^{n}$, see [2].) As is well known (see [3]), the coefficients $v_{j k}$ of the current $d d^{c} v$ for $v \in \operatorname{psh}(\Omega)$ are (locally finite, complex-valued) measures in $\Omega, v_{j j} \geq 0$ and $\left|v_{j k}\right| \leq$ $\sum v_{l l}=\frac{1}{2} \Delta v, j, k=1, \ldots, n$.

STEP 1. The theorem is local, so we can assume that $\Gamma$ is the zero-set of a function $\varrho \in C^{1}(\Omega)$ with $d \varrho \neq 0$ on $\Gamma$. By the Whitney extension theorem, we can also assume that $\varrho \in C^{\infty}(\Omega \backslash \Gamma)$. Set $\lambda_{\varepsilon}=\chi_{\varepsilon} \circ \varrho$, where $\chi_{\varepsilon} \in C^{\infty}(\mathbb{R})$, $0 \leq \chi_{\varepsilon} \leq 1, \chi_{\varepsilon}=0$ in a neighbourhood of 0 and $\chi_{\varepsilon}(t)=1$ for $|t| \geq \varepsilon>0$. Then $d d^{c} u=\mu_{\varepsilon}+\sigma_{\varepsilon}$ where $\mu_{\varepsilon}=\lambda_{\varepsilon} d d^{c} u$ and

$$
\begin{aligned}
\sigma_{\varepsilon} & =\left(1-\lambda_{\varepsilon}\right) d d^{c} u=d\left(\left(1-\lambda_{\varepsilon}\right) d^{c} u\right)+\left(\chi_{\varepsilon}^{\prime} \circ \varrho\right) d \varrho \wedge d^{c} u \\
& =-\left(1-\lambda_{\varepsilon}\right) d^{c} d u=-d^{c}\left(\left(1-\lambda_{\varepsilon}\right) d u\right)-\left(\chi_{\varepsilon}^{\prime} \circ \varrho\right) d^{c} \varrho \wedge d u .
\end{aligned}
$$

STEP 2. As $u \in \operatorname{psh}(\Omega \backslash \Gamma)$ and $\lambda_{\varepsilon}=0$ in a neighbourhood of $\Gamma$, the currents

$$
\mu_{\varepsilon}:=\lambda_{\varepsilon} d d^{c} u=\lambda_{\varepsilon} i \sum u_{j k} d z_{j} \wedge d \bar{z}_{k}
$$

are well defined and positive in $\Omega$. As $u$ is subharmonic in $\Omega$, the measure $\Delta u$ is nonnegative and locally bounded in $\Omega$. As $\left|u_{j k}\right| \leq \Delta u, j, k \leq n$, it follows that

$$
\lim _{\varepsilon \rightarrow 0} \mu_{\varepsilon}=: \mu=i \sum \mu_{j k} d z_{j} \wedge d \bar{z}_{k} \text { exists and is a positive current in } \Omega,
$$

and its coefficients $\mu_{j k}$ are locally finite measures in $\Omega$. By the construction, $\mu$ is carried by $\Omega \backslash \Gamma$, i.e., $\mu_{j k}(E)=0, j, k \leq n$, for any set $E \subset \Gamma$. Moreover, $d d^{c} u=\mu+\sigma$, where

$$
\sigma=\lim _{\varepsilon \rightarrow 0}\left(1-\lambda_{\varepsilon}\right) d d^{c} u
$$

is a current in $\Omega$ supported on $\Gamma$.

Step 3. As $u \in \operatorname{sh}(\Omega)$, the 1-currents $d u, d^{c} u$ have coefficients in $L_{\mathrm{loc}}^{1}(\Omega)$ (this follows obviously from the Riesz decomposition). Thus for every $(2 n-3)$-form $\Psi$ of class $C_{0}^{1}(\Omega)$ (the coefficients belong to $C^{1}(\Omega)$ and have 
compact supports) we see, according to (1), that the value

$$
\begin{aligned}
\left(d d^{c} u, d \varrho \wedge \Psi\right):= & \lim _{\delta \rightarrow 0}\left(d d^{c} u, d \varrho_{\delta} \wedge \Psi\right) \\
= & \lim _{\delta \rightarrow 0}\left(\left(\mu_{\varepsilon}, d \varrho_{\delta} \wedge \Psi\right)+\left(\left(\chi_{\varepsilon}^{\prime} \circ \varrho\right) d \varrho \wedge d^{c} u, d \varrho_{\delta} \wedge \Psi\right)\right. \\
& \left.+\left(\left(1-\lambda_{\varepsilon}\right) d^{c} u, d \varrho_{\delta} \wedge d \Psi\right)\right) \\
= & \left(\mu_{\varepsilon}, d \varrho \wedge \Psi\right)+o_{\varepsilon}(1)=(\mu, d \varrho \wedge \Psi)
\end{aligned}
$$

is well defined. (Here the index $\delta$ means $\delta$-regularization, that is, convolution with a nonnegative function in $C_{0}^{\infty}\left(\mathbb{C}^{n}\right)$ supported in the ball $|z|<\delta$ and having Lebesgue integral 1.)

In the same way, using (2) we find that the values $\left(d d^{c} u, d^{c} \varrho \wedge \Psi\right)$ are well defined and $\left(d d^{c} u, d^{c} \varrho \wedge \Psi\right)=\left(\mu, d^{c} \varrho \wedge \Psi\right)$, which implies that

$$
\begin{aligned}
(\sigma, d \varrho \wedge \Psi) & :=\lim _{\delta \rightarrow 0}\left(\sigma, d \varrho_{\delta} \wedge \Psi\right)=0, \\
\left(\sigma, d^{c} \varrho \wedge \Psi\right) & :=\lim _{\delta \rightarrow 0}\left(\sigma, d^{c} \varrho_{\delta} \wedge \Psi\right)=0 .
\end{aligned}
$$

STEP 4. Let $\varphi \in C_{0}(\Omega), \varphi \geq 0$, and let $\omega=\frac{1}{4} d d^{c}|z|^{2}$ be the fundamental form in $\mathbb{C}^{n}$. Then

$$
\left(d d^{c} u, \varphi \omega^{n-1}\right):=\lim _{\delta \rightarrow 0}\left(d d^{c} u, \varphi_{\delta} \omega^{n-1}\right)=4(n-1) !(\Delta u, \varphi) \geq 0,
$$

hence,

$$
\lim _{\delta \rightarrow 0}\left(\mu+\sigma,\left(1-\lambda_{\varepsilon}\right) \varphi_{\delta} \omega^{n-1}\right)=\left(\mu,\left(1-\lambda_{\varepsilon}\right) \varphi \omega^{n-1}\right)+\lim _{\delta \rightarrow 0}\left(\sigma, \varphi_{\delta} \omega^{n-1}\right)
$$

is nonnegative. As $\mu$ is carried by $\Omega \backslash \Gamma$ and $1-\lambda_{\varepsilon} \rightarrow 0$ there, we can pass to the limit as $\varepsilon \rightarrow 0$ and obtain

$$
\left(\sigma, \varphi \omega^{n-1}\right):=\lim _{\delta \rightarrow 0}\left(\sigma, \varphi_{\delta} \omega^{n-1}\right) \geq 0 .
$$

STEP 5. Let $\Phi$ be an arbitrary $(n-1, n-1)$-form of class $C_{0}^{\infty}(\Omega)$ and $\Phi_{\tau}$ be its projection onto the complex tangent planes $T_{z}^{c}$ to the levels $\{\varrho(z)=$ const $\}$. (Representing $\Phi=\sum_{j, k=1}^{n} \Phi_{j k} \prod_{\alpha \neq j} d z_{\alpha} \wedge \prod_{\beta \neq k} d z_{\beta}$ and assuming that $T_{a}^{c}=\left\{z_{n}=0\right\}$, which can be done by a unitary transform, we see that $\left.\Phi_{\tau}\right|_{a}$ is the same sum from 1 to $n-1$ at the point $a$.) It is obvious that $\Phi_{\tau}$ is a $(2 n-2)$-form of class $C_{0}(\Omega)$. Moreover, $\Phi=\Phi_{\tau}+\Phi_{\nu}$, where $\Phi_{\nu}$ is in the same class and $\Phi_{\nu}\left(\tau_{1} \wedge \ldots \wedge \tau_{n-1}\right)=0$ for any vector fields $\tau_{1}, \ldots, \tau_{n-1}$ which are complex orthogonal to $\nabla \varrho$.

By the Wirtinger theorem, the restrictions $\omega^{n-1} /(n-1) ! \mid T_{z}^{c}$ coincide with the usual volume form on the planes $T_{z}^{c}$, hence

$$
\Phi\left|T_{z}^{c}=\Phi_{\tau}\right| T_{z}^{c}=\varphi \omega^{n-1} \mid T_{z}^{c}
$$

for some $\varphi \in C_{0}(\Omega)$. Moreover, $\varphi \geq 0$ if the form $\Phi$ is positive (because $T_{z}^{c}$ are complex planes). 
Decomposing $\omega^{n-1}=\left(\omega^{n-1}\right)_{\tau}+\left(\omega^{n-1}\right)_{\nu}$, we obtain, for arbitrary $\Phi$, the decomposition $\Phi=\varphi \omega^{n-1}+\Phi_{0}$, where $\Phi_{0}$ is orthogonal to $T_{z}^{c}$ in the sense that $\Phi_{0}\left(\tau_{1} \wedge \ldots \wedge \tau_{n-1}\right)=0$ for any fields $\tau_{j}$ as above. As $d \varrho, d^{c} \varrho$ constitute a basis of 1-covectors annihilating all such $\tau_{j}$, the form $\Phi_{0}$ is represented as a sum $d \varrho \wedge \Psi_{1}+d^{c} \varrho \wedge \Psi_{2}$ with continuous $\Psi_{1}, \Psi_{2}$ in $\Omega$. Finally, we have the decomposition

$$
\Phi=\varphi \omega^{n-1}+\Phi_{0}=\varphi \omega^{n-1}+d \varrho \wedge \Psi_{1}+d^{c} \varrho \wedge \Psi_{2},
$$

where all the terms belong to the class $C_{0}(\Omega)$, and $\varphi \geq 0$ if $\Phi$ is a positive form of bidegree $(n-1, n-1)$.

STEP 6. Let now

$$
\Phi^{\delta}:=\varphi_{\delta} \omega^{n-1}+\Phi_{0}^{\delta}:=\varphi_{\delta} \omega^{n-1}+d \varrho \wedge\left(\Psi_{1}\right)_{\delta}+d^{c} \varrho \wedge\left(\Psi_{2}\right)_{\delta}
$$

for a positive $\Phi$ of class $C_{0}^{\infty}(\Omega)$ and bidegree $(n-1, n-1)$. Then

$$
\left(d d^{c} u, \Phi^{\delta}\right)=\left(\mu, \Phi^{\delta}\right)+\left(\sigma, \varphi_{\delta} \omega^{n-1}\right),
$$

according to (3) and $\left(3^{\prime}\right)$. As $\varphi \geq 0$, we have $\left(\sigma, \varphi_{\delta} \omega^{n-1}\right) \geq 0$ by (4). Thus

$$
\left(d d^{c} u, \Phi\right)=\lim _{\delta \rightarrow 0}\left(d d^{c} u, \Phi^{\delta}\right) \geq \lim _{\delta \rightarrow 0}\left(\mu, \Phi^{\delta}\right)=(\mu, \Phi) \geq 0,
$$

as $\Phi$ has bidegree $(n-1, n-1)$ and $\mu, \Phi$ are positive.

The theorem is proved.

\section{References}

[1] P. Blanchet, On removable singularities of subharmonic and plurisubharmonic functions, Complex Variables 26 (1995), 311-322.

[2] R. Harvey, Holomorphic chains and their boundaries, in: Proc. Sympos. Pure Math. 30, Part 1, Amer. Math. Soc., 1977, 307-382.

[3] P. Lelong, Fonctions plurisousharmoniques et formes différentielles positives, Gordon and Breach, New York, 1968.

Steklov Mathematical Institute

Gubkin st. 8

Moscow, Russia

E-mail: chirka@mi.ras.ru

Reçu par la Rédaction le 30.1.2002

Révisé le 8.4.2002 